\title{
A novel preoperative predictive model of 90-day mortality after liver resection for huge hepatocellular carcinoma
}

\author{
Yue Yin ${ }^{1,2 \#}$, Jian-Wen Cheng ${ }^{1 \#}$, Fei-Yu Chen ${ }^{1 \#}$, Xu-Xiao Chen ${ }^{1}$, Xin Zhang ${ }^{1}$, Ao Huang ${ }^{1}$, De-Zhen Guo ${ }^{1}$, \\ Yu-Peng Wang ${ }^{1}$, Ya Cao ${ }^{3}$, Jia Fan ${ }^{1,4,5}$, Jian Zhou ${ }^{1,4,5}$, Xin-Rong Yang ${ }^{1}$ \\ ${ }^{1}$ Department of Liver Surgery and Transplantation, Liver Cancer Institute, Zhongshan Hospital, Fudan University; Key Laboratory of \\ Carcinogenesis and Cancer Invasion (Fudan University), Ministry of Education; Shanghai Key Laboratory of Organ Transplantation, Zhongshan \\ Hospital, Fudan University, Shanghai, China; ${ }^{2}$ Department of General Surgery, Shanghai Ninth People's Hospital, Shanghai Jiao Tong University \\ School of Medicine, Shanghai, China; ${ }^{3}$ Cancer Research Institute, Central South University; Key Laboratory of Carcinogenesis and Cancer Invasion, \\ Ministry of Education, Changsha, China; ${ }^{4}$ Institute of Biomedical Sciences, Fudan University, Shanghai, China; ${ }^{5}$ State Key Laboratory of Genetic \\ Engineering, Fudan University, Shanghai, China \\ Contributions: (I) Conception and design: XR Yang, J Zhou; (II) Administrative support: None; (III) Provision of study materials or patients: None; (IV) \\ Collection and assembly of data: All authors; (V) Data analysis and interpretation: Y Yin, JW Cheng, FY Chen; (VI) Manuscript writing: All authors; \\ (VII) Final approval of manuscript: All authors. \\ "These authors contributed equally to this work. \\ Correspondence to: Xin-Rong Yang, MD, PhD; Jian Zhou, MD, PhD. Department of Liver Surgery and Transplantation, Liver Cancer Institute, \\ Zhongshan Hospital, Fudan University, 136 Yixueyuan Road, Shanghai 200032, China. \\ Email: yang.xinrong@zs-hospital.sh.cn; zhou.jian@zs-hospital.sh.cn.
}

Background: Hepatectomy for huge hepatocellular carcinoma (HCC) (diameter $\geq 10 \mathrm{~cm}$ ) is characterized by high mortality. This study aimed to establish a preoperative model to evaluate the risk of postoperative 90-day mortality for huge HCC patients.

Methods: We retrospectively enrolled 1,127 consecutive patients and prospectively enrolled 93 patients with huge HCC who underwent hepatectomy (training cohort, $n=798$; validation cohort, $n=329$; prospective cohort, $\mathrm{n}=93$ ) in our institute. Based on independent preoperative predictors of 90-day mortality, we established a logistic regression model and visualized the model by nomogram.

Results: The 90-day mortality rates were $9.6 \%, 9.2 \%$, and $10.9 \%$ in the training, validation, and prospective cohort. The $\alpha$-fetoprotein (AFP) level, the prealbumin levels, and the presence of portal vein tumor thrombosis (PVTT) were preoperative independent predictors of 90-day mortality. A logistic regression model, AFP-prealbumin-PVTT score (APP score), was subsequently established and showed good performance in predicting 90-day mortality (training cohort, AUC $=0.87$; validation cohort, AUC $=0.91$; prospective cohort, AUC $=0.93)$. Using a cut-off of -1.96 , the model could stratify patients into low risk ( $\leq-1.96)$ and high risk (>-1.96) with different 90 -day mortality rates $(\sim 30 \% v s . \sim 2 \%)$. Furthermore, the predictive performance for 90-day mortality and overall survival was significantly superior to the Child-Pugh score, the model of end-stage liver disease (MELD) score, and the albumin-bilirubin (ALBI) score.

Conclusions: The APP score can precisely predict postoperative 90-day mortality as well as long-term survival for patients with huge HCC, assisting physician selection of suitable candidates for liver resection and improving the safety and efficacy of surgical treatment.

Keywords: Hepatectomy; huge hepatocellular carcinoma; 90-day mortality; prediction

Submitted Dec 06, 2020. Accepted for publication Feb 28, 2021.

doi: 10.21037/atm-20-7842

View this article at: http://dx.doi.org/10.21037/atm-20-7842 


\section{Introduction}

Hepatocellular carcinoma (HCC) is one of the most prevalent malignancies and the second-leading cause of cancer-related deaths worldwide (1). Huge HCC is defined as $\mathrm{HCC}$ with a maximum tumor diameter greater than $10 \mathrm{~cm}$ and accounts for $10-20 \%$ of newly developed HCC cases (2). Surgical resection is considered a potential curative option for these patients and has been reported to provide a favorable overall survival (OS) in some patients $(3,4)$. Although mortality after liver resection has been dramatically reduced in recent years with advances in surgical techniques and perioperative management (5-7), the postoperative mortality rate of hepatectomy for huge HCC, ranging from $2.7 \%$ to $18.1 \%$, is still much higher than the routine liver resection $(\sim 1 \%)(8-10)$. As the natural history for a patient with advanced HCC is approximately 3-6 months after diagnosis (11), death within 3 months after hepatectomy is regarded as "futile liver resection," which should be avoided because it provides no survival benefit $(8,12)$. Therefore, there is an urgent need to identify patients with huge HCC who have a high risk of postoperative 90 -day mortality to optimize the candidate selection for liver resection and avoid futile liver resection (12).

Several models, such as the Child-Pugh score (13), the model for end-stage liver disease (MELD) score $(14,15)$, and the albumin-bilirubin (ALBI) score (16) have been widely applied to evaluate the risk of postoperative outcome for patients with HCC (17-22). However, none of these models were specifically developed to predict the posthepatectomy mortality for HCC. Some other established prediction models are based on intraoperative data or postoperative liver function (23-27), which could not help preoperative decision-making. Furthermore, some preoperative models include patients undergoing hepatectomy for various indications, making the model lack representativeness of a particular patient group (28). Currently, a model to preoperatively evaluate postoperative 90 -day mortality in patients with huge HCC is still lacking.

In this study, we aimed to identify the preoperative available parameters that independently influenced postoperative 90 -day mortality in patients with huge HCC undergoing hepatectomy and to develop a simple, preoperative risk assessment model for precisely predicting postoperative 90 -day mortality for those patients with huge HCC, which may help surgeon select suitable candidates to improve the safety and efficacy for liver resection. We present the following article following the STROBE reporting checklist (available at http://dx.doi.org/10.21037/ atm-20-7842).

\section{Methods}

\section{Patient enrollment}

A total of 1,127 consecutive huge HCC patients who underwent liver resection were retrospectively enrolled and divided into two independent cohorts (training cohort, $\mathrm{n}=798$, January 2007 to December 2012 and validation cohort, $\mathrm{n}=329$, January 2013 to December 2014). Furthermore, another independent cohort (prospective cohort, n=93) was prospectively enrolled between March 2019 and March 2020. The inclusion criteria were as follows: (I) liver resection in Zhongshan hospital with complete removal of the tumor; (II) HCC with a maximum tumor diameter greater than or equal to $10 \mathrm{~cm}$. The exclusion criteria were as follows: (I) preoperative distant metastasis; (II) multi-organ removal (except for cholecystectomy).

Ethical approval for the use of patient information was obtained from the Zhongshan Hospital Research Ethics Committee (No. B2021-017R). Informed consent was obtained from all patients. The study was conducted in accordance with the Declaration of Helsinki (as revised in 2013).

\section{Perioperative management and follow-up}

Contrast-enhanced magnetic resonance imaging (MRI) or computed tomography (CT) were routinely performed preoperatively. Solid lesions within the portal vein observed during all phases of intravenous contrast-enhanced CT or MRI, especially with the enhancement of contrast during the arterial phase and washout during the portal venous phase of the procedure, were regarded as portal vein tumor thrombosis (PVTT) (29). The spleen over $9.76 \mathrm{~cm}$ in length by the preoperative radiological finding was regarded as splenomegaly (30). Portal hypertension was defined as the presence of either esophageal varices or splenomegaly with a platelet count less than $10^{9} / \mathrm{L}$ (31). CT volumetry was used to evaluate the volume of the future liver remnant (FLR) in cases with an apparent inadequate FLR. The estimated standard liver volume (SLV) was calculated based on the Urata formula (32). A hepatectomy was only recommended when patients had sufficient future liver remnant. For the patients with a background of liver cirrhosis diagnosed by the MRI, an FLR/SLV ratio $>40 \%$ was considered to be 


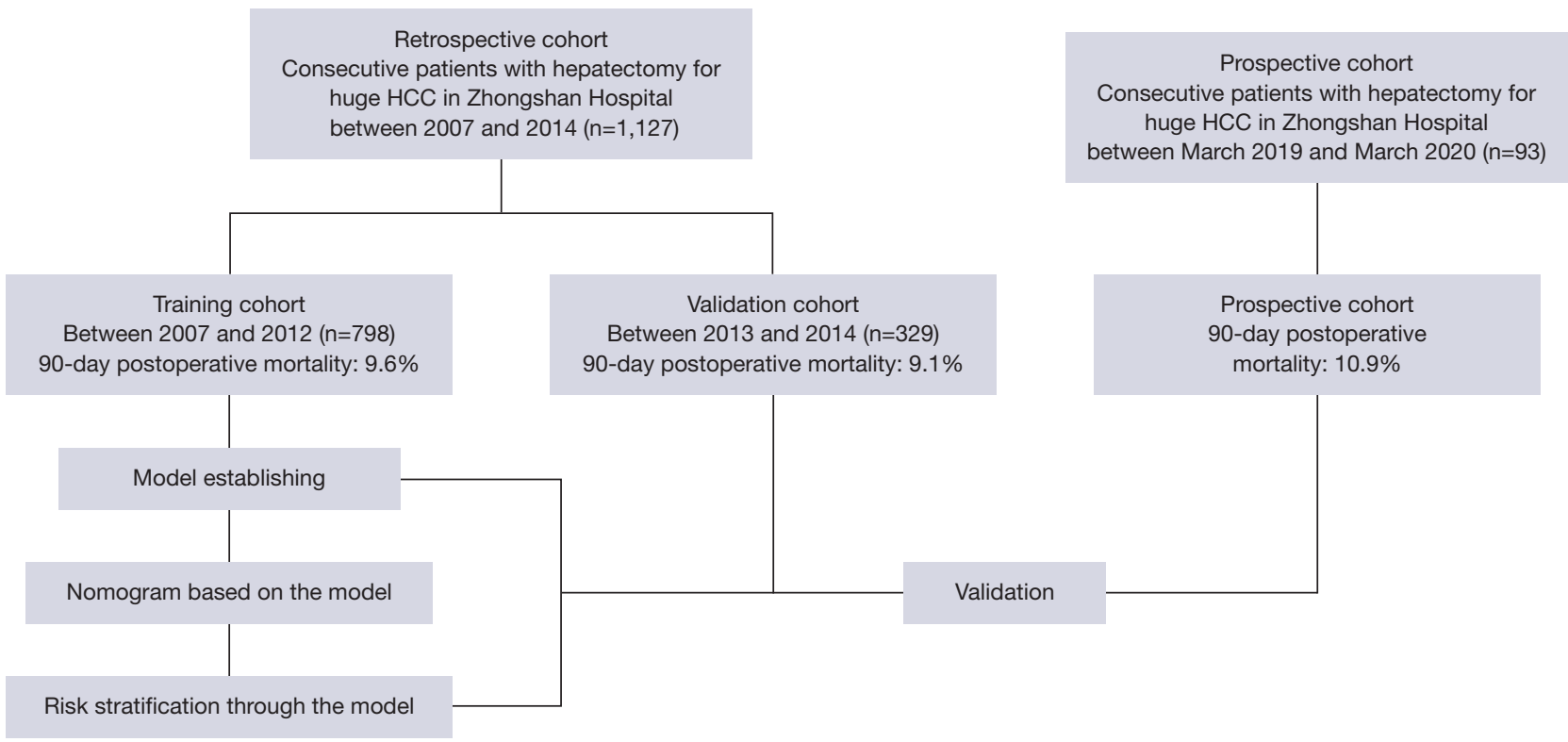

Figure 1 Flow chart for the study design and patients enrollment.

safe for operation, whereas a ratio $>30 \%$ was considered sufficient in the patients without liver cirrhosis (33). The major hepatectomy was defined as liver resection with more than 3 segments (34). The preoperative Child-Pugh (13), MELD (14), and ALBI (16) scores were also calculated as previously described.

After surgery, regular hepatoprotective dosages and albumin supplementation were administered. Antiviral agents (entecavir) were recommended for all patients with hepatitis B infection. Postoperative complications were categorized according to the Clavien-Dindo Classification (35). Postoperative follow-up examinations were performed monthly for 3 months and every 3 months thereafter. Patients with suitable liver function were recommended to undergo one to three courses of adjuvant transcatheter arterial chemoembolization (TACE; with doxorubicin, cisplatin, 5-fluorouracil, and iodized oil) (36); sorafenib was recommended for patients with PVTT or microvascular invasion. The follow-up was terminated in April 2019 for two retrospective cohorts and in June 2020 for the prospective cohort. The main end-point of this study was 90 -day mortality.

\section{Statistical analysis}

Statistical analyses were performed using R software (version 3.6.1; https://www.r-project.org/) and GraphPad Prism software (version 7.0; GraphPad Software Inc., San Diego, CA, USA). Categorical variables were compared using the $\chi^{2}$ test or Fisher's exact test. A nomogram was created based on the results of the multivariate logistic analysis using the rms package. The model was validated using the bootstrap resampling technique in the training and validation cohort. Comparisons of the newly developed model with ChildPugh, MELD, and ALBI scores were carried out using the receiver operating characteristic curve (ROC curve) and the area under the curve (AUC). The ROC curve and the AUC were derived to assess the performance of the model in the validation and prospective cohort. OS was compared using the log-rank test, and risk factors for survival were identified using the Cox regression method. A two-tailed $\mathrm{P}$ value of less than 0.05 was considered significant.

\section{Results}

\section{Patient demographics}

A total of 1,127 patients were enrolled in this study and divided into the training cohort $(\mathrm{n}=798)$ and the validation cohort $(n=329)$. Meanwhile, an additional cohort of 93 patients with huge HCC resection (prospective cohort) was prospectively enrolled for further validation. The study design is shown in Figure 1. The characteristics of the training, validation, and prospective cohorts are summarized 
in Table 1 and Table S1. The postoperative 90-day mortality rate was $9.6 \%(77 / 798)$ in the training cohort, $9.2 \%$ (30/329) in the validation cohort, and $10.9 \%(10 / 93)$ in the prospective cohort, respectively. All enrolled patients were categorized according to the Child-Pugh A classification. The majority of patients had hepatitis B: $76.1 \%$ in the training cohort, $77.8 \%$ in the validation cohort, and $73.1 \%$ in the prospective cohort.

\section{Preoperative risk factors for postoperative 90-day mortality in the training cohort}

The preoperative available clinical indices were used to build up the model. The univariate logistic regression analysis of the training cohort showed that the maximum tumor diameter, PVTT status, prealbumin level, platelet count, total bilirubin level, albumin level, prothrombin time, AFP level, the extent of tumor differentiation, and portal hypertension were statistically significant (Table 2). Additionally, the logistic regression analysis revealed that PVTT status was associated with serum prealbumin levels $(\mathrm{P}=0.010)$. We included all parameters with a $\mathrm{P}$ value less than 0.1 in the multivariate logistic regression analysis, which revealed that preoperative AFP level, prealbumin level, and PVTT status were independent predictors of 90-day mortality after surgery (Table 3).

\section{Construction of the APP score for predicting postoperative 90-day mortality}

A logistic regression model used these three parameters (prealbumin level, the presence or absence of PVTT, and AFP level) was constructed and derived a linear predictor equation as follows: Linear predictor $=($ PVTT $)$ $\times$ prealbumin $\times 17.17+\mathrm{AFP} \times 2.945 \times 10^{-5}+$ prealbumin $\times$ (-26.23), where prealbumin is in $\mathrm{g} / \mathrm{L}, \mathrm{AFP}$ is in $\mathrm{ng} / \mathrm{mL}$, and PVTT was assigned a " 1 " when present or " 0 " when absent, as determined by preoperative radiological findings. The linear predictor model was named "the AFP-prealbuminPVTT score (APP score)" and visualized using a nomogram (Figure 2).

The AUC of the APP score in predicting 90-day mortality was 0.87 (95\% CI, 0.84-0.91) in the training cohort, which was significantly higher than the AUC of the Child-Pugh score $(0.55$; 95\% CI, 0.48-0.62, $\mathrm{P}<0.001)$, the MELD score $(0.64 ; 95 \% \mathrm{CI}, 0.57-0.70, \mathrm{P}<0.001)$, and the ALBI score $(0.64 ; 95 \%$ CI, 0.57-0.70, $\mathrm{P}<0.001)$ (Figure $3 A$ ). Meanwhile, the optimal cut-off value for the APP score was proposed to be -1.96 using the maximum Youden index, with a sensitivity of $80.5 \%$ and a specificity of $80.8 \%$ in the training cohort (Figure $3 A$ ). According to this cut-off value, the APP score could stratify patients in the training cohort into two risk groups for 90-day mortality: low risk $(\leq-1.96$, $\mathrm{n}=598)$ and high risk $(>-1.96, \mathrm{n}=200)$. The high-risk group had a significantly higher 90 -day mortality rate than the low-risk group ( $31.0 \%$ vs. $2.5 \%, \mathrm{P}<0.001$, Figure $3 B)$.

\section{Validation of the APP score in predicting 90-day mortality after bepatectomy}

The performance of the APP score in predicting postoperative 90 -day mortality was further evaluated. The AUC of the APP score was 0.91 (95\% CI, 0.86-0.95) in the validation cohort, which was also superior to the Child-Pugh score $(0.59 ; 95 \%$ CI, $0.48-0.71 ; \mathrm{P}<0.001)$, the MELD score (0.61; 95\% CI, 0.50-0.73; $\mathrm{P}<0.001)$, and the ALBI score (0.66; 95\% CI, 0.56-0.77; $\mathrm{P}<0.001$ ) (Figure 3C). The higher incidence of 90-day mortality was also observed in the highrisk group compared to the low-risk group (validation cohort: $32.0 \%$ vs. $2.4 \%, \mathrm{P}<0.001$; Figure $3 D$ ). In the prospective cohort, the APP score (AUC $=0.93,95 \%$ CI, 0.86-1.00) showed a consistent predictive ability with a higher AUC than the Child-Pugh score $(0.53 ; 95 \%$ CI, 0.33-0.73; $\mathrm{P}<0.001)$, the MELD score $(0.58 ; 95 \% \mathrm{CI}, 0.41-0.74 ; \mathrm{P}<0.001)$ and the ALBI score (0.76; 95\% CI, 0.58-0.93; $\mathrm{P}=0.037$ ) (Figure 3E). Using the same cut-off value of -1.96 , the sensitivity and the specificity of the APP score in predicting 90-day mortality were $80.0 \%$ and $82.9 \%$ in the validation cohort, and $81.6 \%$ and $90.0 \%$ in the prospective cohort, respectively. In the prospective cohort, the 90-day mortality rate in the highrisk group was also higher than the low-risk group $37.5 \%$ vs. $1.4 \%, \mathrm{P}<0.001$; Figure $3 F)$.

Nevertheless, calibration using bootstrap sampling showed good agreement among the apparent curve, the bias-corrected curve, and the ideal curve in the training and validation cohort (Figure S1). The subgroup analysis in the training and validation cohort indicated that the patients with a higher APP score always had a higher mortality rate regardless of the liver status (cirrhosis), preoperative treatment (TACE), and surgical strategy (anatomical or non-anatomical hepatectomy) (Table S2).

\section{Performance of the APP score in predicting long-term survival for patients with buge HCC after hepatectomy}

The median follow-ups were 70.3 months in the training 
Table 1 Clinicopathologic characteristics of the training, validation, and prospective cohorts

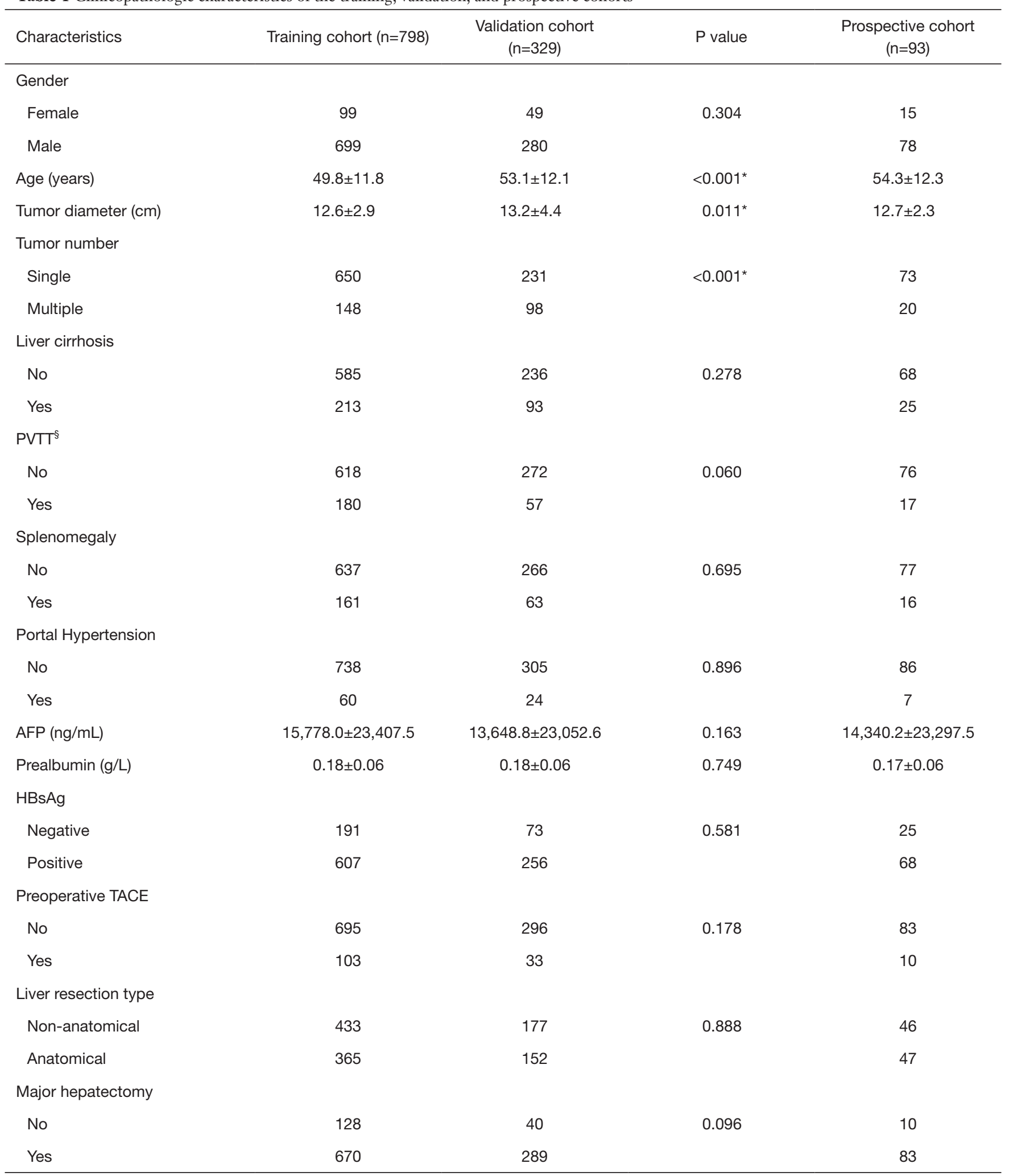

Table 1 (continued) 
Table 1 (continued)

\begin{tabular}{|c|c|c|c|c|}
\hline Characteristics & Training cohort $(n=798)$ & $\begin{array}{l}\text { Validation cohort } \\
\qquad(\mathrm{n}=329)\end{array}$ & $P$ value & $\begin{array}{l}\text { Prospective cohort } \\
\qquad(n=93)\end{array}$ \\
\hline \multicolumn{5}{|c|}{ Clavien-Dindo classification } \\
\hline None or $<$ III & 663 & 280 & 0.403 & 80 \\
\hline$\geq \mathrm{III}$ & 135 & 49 & & 13 \\
\hline ALBI score & $-2.65 \pm 0.37$ & $-2.63 \pm 0.35$ & 0.451 & $-2.85 \pm 0.37$ \\
\hline
\end{tabular}

$\S$, among all the patients, 3 patients ( 2 in the training cohort and 1 in the validation cohort) had histologically proved tumor thrombosis in small branches of portal vein near the tumor (Vp1) but could not be detected by preoperative imaging examinations. ${ }^{*}, \mathrm{P}<0.05$. These 3 patients were not included in the PVTT group. PVTT, portal vein tumor thrombosis; AFP, $\alpha$-fetoprotein; HBsAg, hepatitis B surface antigen; MELD, model of end-stage liver disease; ALBI, albumin-bilirubin.

Table 2 Univariate logistic regression analysis to identify the risk factors for the postoperative 90-day mortality for huge HCC

\begin{tabular}{|c|c|c|c|}
\hline Clinical feature & OR & $95 \% \mathrm{Cl}$ & $P$ value \\
\hline Age (year) & 0.980 & $0.961-0.999$ & 0.053 \\
\hline HBsAg (positive: negative) & 1.335 & $0.741-2.406$ & 0.337 \\
\hline Diabetes mellitus (yes: no) & 0.770 & $0.100-10.32$ & 0.986 \\
\hline Tumor number (multiple: single) & 1.025 & $0.933-1.126$ & 0.606 \\
\hline PVTT (yes: no) & 9.636 & 5.764-16.109 & $<0.001^{*}$ \\
\hline GGT (U/L) & 1.001 & $0.999-1.003$ & 0.275 \\
\hline Prealbumin $(g / L)$ & $<0.001$ & $0.000-0.001$ & $<0.001^{*}$ \\
\hline Total bilirubin ( $\mu \mathrm{mol} / \mathrm{L})$ & 1.02 & $1.004-1.036$ & $0.013^{*}$ \\
\hline Albumin (g/L) & 0.941 & $0.888-0.997$ & $0.039^{*}$ \\
\hline Prothrombin time (s) & 1.455 & $1.210-1.750$ & $<0.001^{\star}$ \\
\hline Creatine $(\mu \mathrm{mol} / \mathrm{L})$ & 1.007 & $0.996-1.018$ & 0.205 \\
\hline $\operatorname{AFP}(n g / m L)$ & 1.000 & $1.000-1.001$ & $<0.001^{*}$ \\
\hline Tumor differentiation (III-IV: I-II) & 2.592 & $1.610-4.171$ & $<0.001^{\star}$ \\
\hline Splenomegaly (yes: no) & 1.197 & $0.607-2.360$ & 0.604 \\
\hline
\end{tabular}

*, P<0.05. HCC, hepatocellular carcinoma; 95\% Cl, 95\% confidential interval; HBsAg, hepatitis B surface antigen; PVTT, portal vein tumor thrombosis; GGT, $\gamma$-glutamyl transpeptidase; ALT, Alanine Aminotransferase; AFP, $\alpha$-fetoprotein. 
Table 3 Multivariate logistic regression analysis to identify the risk factors for the postoperative 90-day mortality for huge HCC

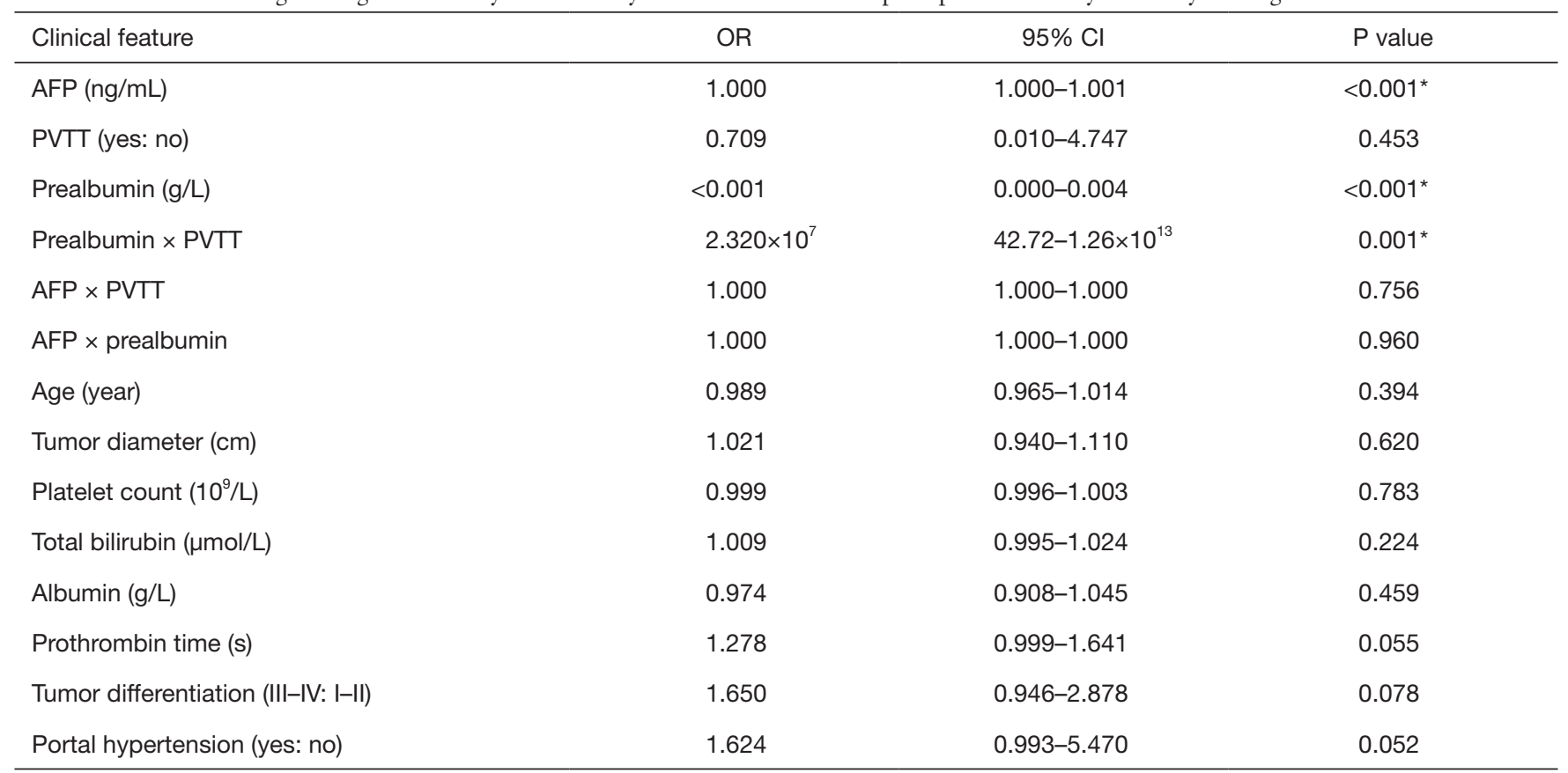

*, P<0.05. HCC, hepatocellular carcinoma; $95 \% \mathrm{Cl}$, 95\% confidential interval; PVTT, portal vein tumor thrombosis; AFP, $\alpha$-fetoprotein.

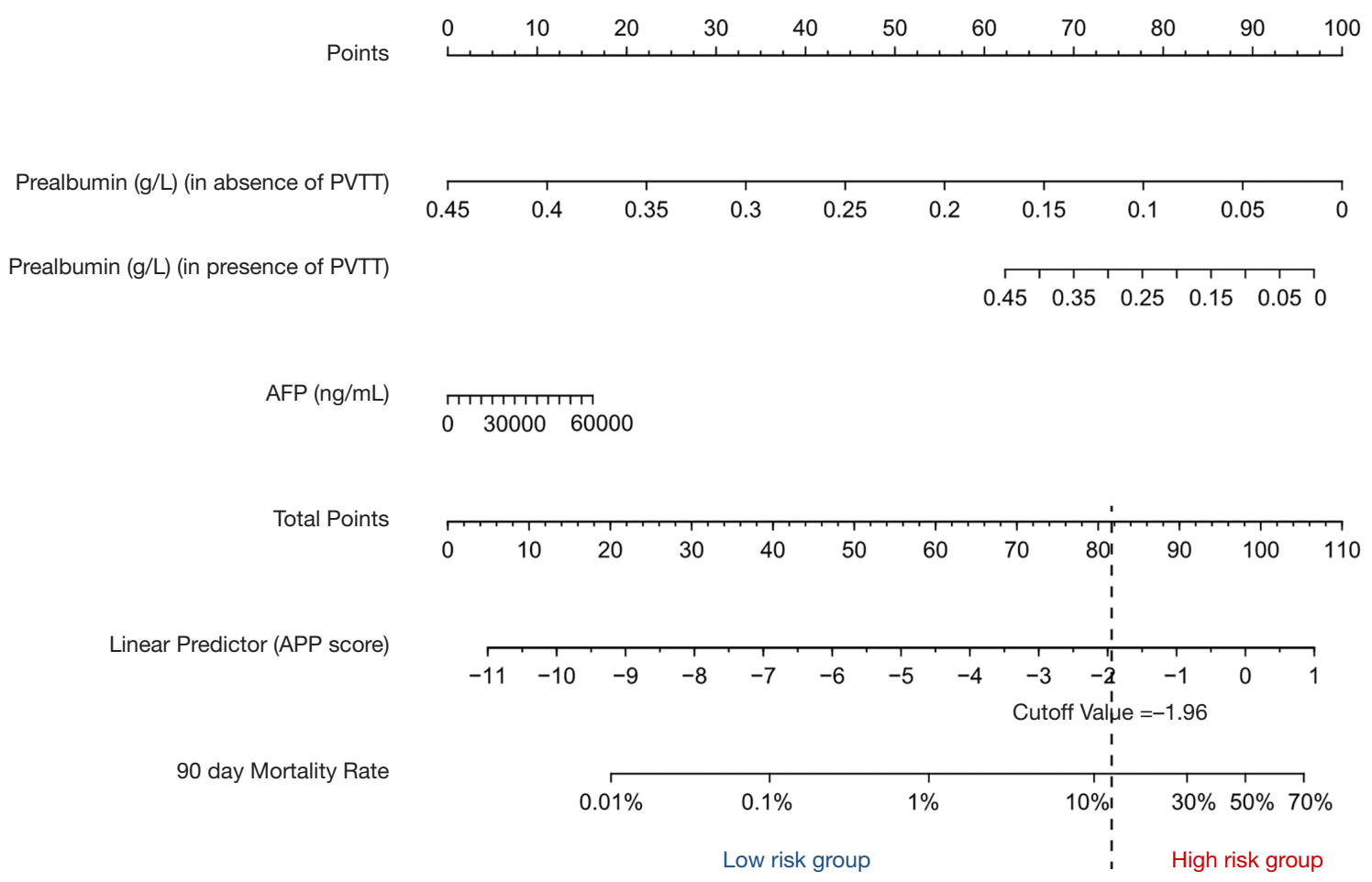

Figure 2 Nomogram for predicting the postoperative 90-day mortality for huge HCC patients. The points of each variable in rows 2 to 4 are added up to the total points presented on the scale in row 5 , which corresponds to the linear predictor in row 6 and the 90 -day mortality rate in row 7. We named the linear predictor as the AFP-Prealbumin-PVTT score (the APP score), and the patients were stratified into lowrisk ( $\leq-1.96)$ and high risk (>-1.96). 

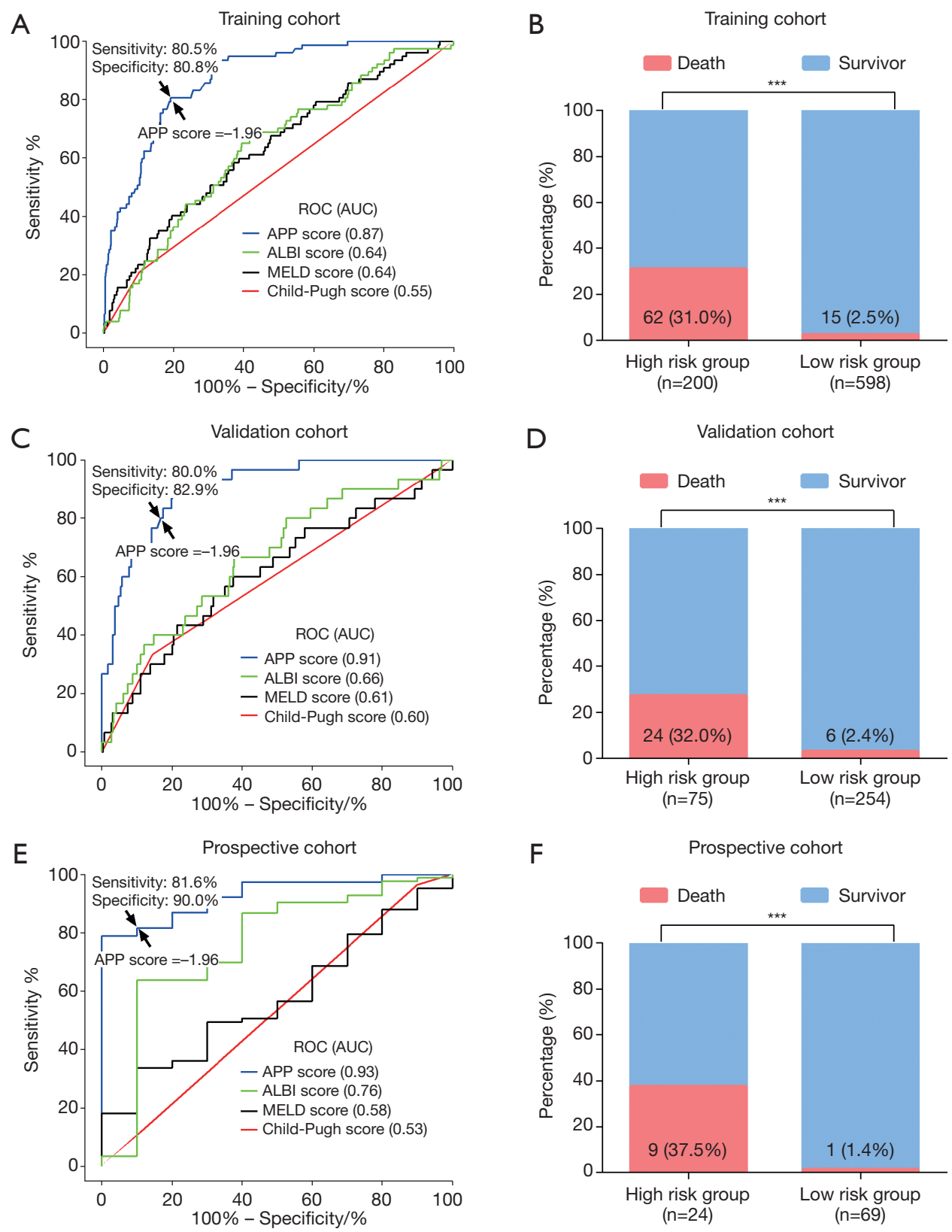

Figure 3 The predictive performance of the APP score for the postoperative 90-day mortality for huge HCC. (A) In the training cohort, the AUC of APP score was significantly larger than the ALBI, MELD score, and Child-Pugh score (all P<0.001). The sensitivity of $80.5 \%$ and the specificity of $80.8 \%$ was achieved when using the cutoff (APP $=-1.96$, arrow) derived from the maximum of Youden index. (B) The patients in the high-risk group (APP score >-1.96) had a significantly higher 90-day mortality rate than the low-risk group (APP score $\leq-1.96)$ in the training cohort $(31.0 \%$ vs. $2.5 \%, \mathrm{P}<0.001)$. (C) In the validation cohort, the AUC of APP score was significantly higher than the ALBI score, MELD score and Child-Pugh score (all $\mathrm{P}<0.001$ ). With the cutoff derived from the training cohort (arrow), the sensitivity was $80.0 \%$ and the specificity was $82.9 \%$. (D) The patients in the high-risk group (APP score >-1.96) had a significant higher 90 -day mortality rate than the low-risk group (APP score $\leq-1.96)$ in the validation cohort, $(32.0 \%$ vs. $2.4 \%, \mathrm{P}<0.001)$. (E) In the prospective cohort, the AUC of APP score was significantly higher than the ALBI score, MELD score and Child-Pugh score (all P<0.001). With the cutoff derived from the training cohort (arrow), the sensitivity was $81.6 \%$ and the specificity was $90.0 \%$. (F) The patients in the high-risk group (APP score $>-1.96$ ) had a significantly higher 90 -day mortality rate than the low-risk group (APP score $\leq-1.96)$ in the prospective cohort $(37.5 \%$ vs. $1.4 \%, \mathrm{P}<0.001)$. ***, $\mathrm{P}<0.001$. 

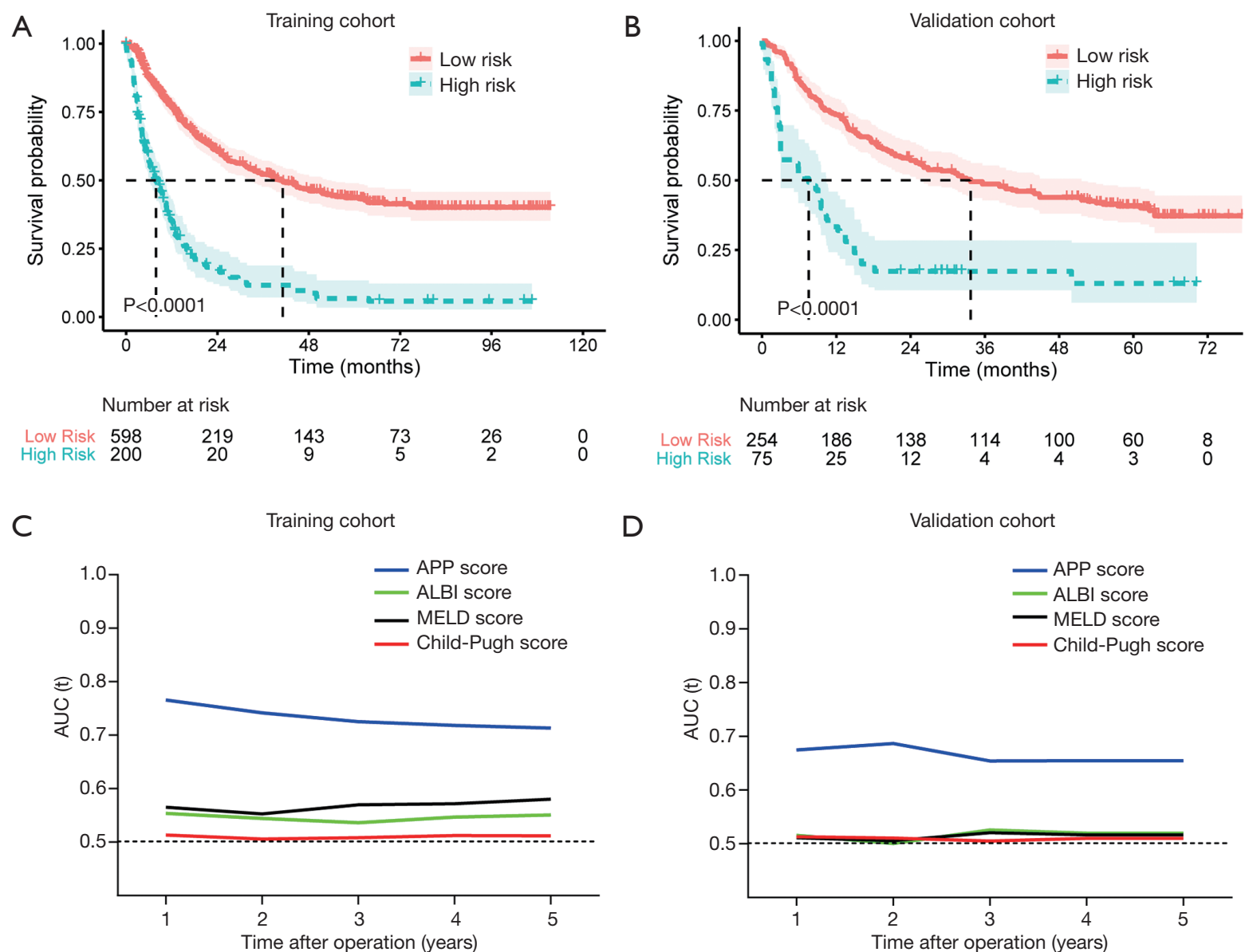

Figure 4 Performance of the nomogram in predicting overall survival for huge HCC undergoing resection. The overall survival (OS) of the patients in the high-risk group (APP score $>-1.96$ ) was significantly longer than the low-risk group (APP score $\leq-1.96$ ) in both (A) the training cohort $(\mathrm{P}<0.001)$, and $(\mathrm{B})$ validation cohort $(\mathrm{P}<0.001)$. Time-dependent AUC showed the performance of the APP score, ChildPugh score, MELD score and ALBI score in predicting OS in (C) the training cohort, and (D) the validation cohort. The AUC of APP score was significantly higher than the ALBI score, MELD score, and Child-Pugh score in predicting 1-, 2-, 3-, 4-, and 5-year OS in the two cohorts (all $\mathrm{P}<0.001)$.

cohort (interquartile range: $35.2-104.3$ months) and 52.6 months in the validation cohort (interquartile range: 44.1-67.9). Using a threshold of -1.96 , the APP score stratified patients into two separate groups with significantly different OS rates in training (median OS: $7.87 v s$. 41.2 months) and validation cohort (median OS: $7.53 \mathrm{vs}$. 33.7 months) (Figure $4 A, B)$. In the multivariate analysis, the APP score was identified as an independent prognostic factor for $\mathrm{OS}$ in both the training $(\mathrm{HR}=3.78, \mathrm{P}<0.001)$ and validation cohorts $(\mathrm{HR}=2.54, \mathrm{P}<0.001)$ (Table 4). In addition, the AUCs of the APP score in predicting OS were significantly higher than those of the Child-Pugh, MELD, and ALBI scores in both the training and validation cohorts
(Figure 4C,D).

\section{Discussion}

Due to advances in surgical technique, preoperative evaluation, and perioperative management, surgical indications for huge HCC tumors have been expanded (7). However, there is still no consensus on the criteria to select patients with huge HCC for surgical treatment to avoid postoperative mortality (37). How to preoperatively evaluate the risk of postoperative 90-day mortality precisely is thus essential to further improve the safety and efficacy of the liver resection for huge HCC (8). 


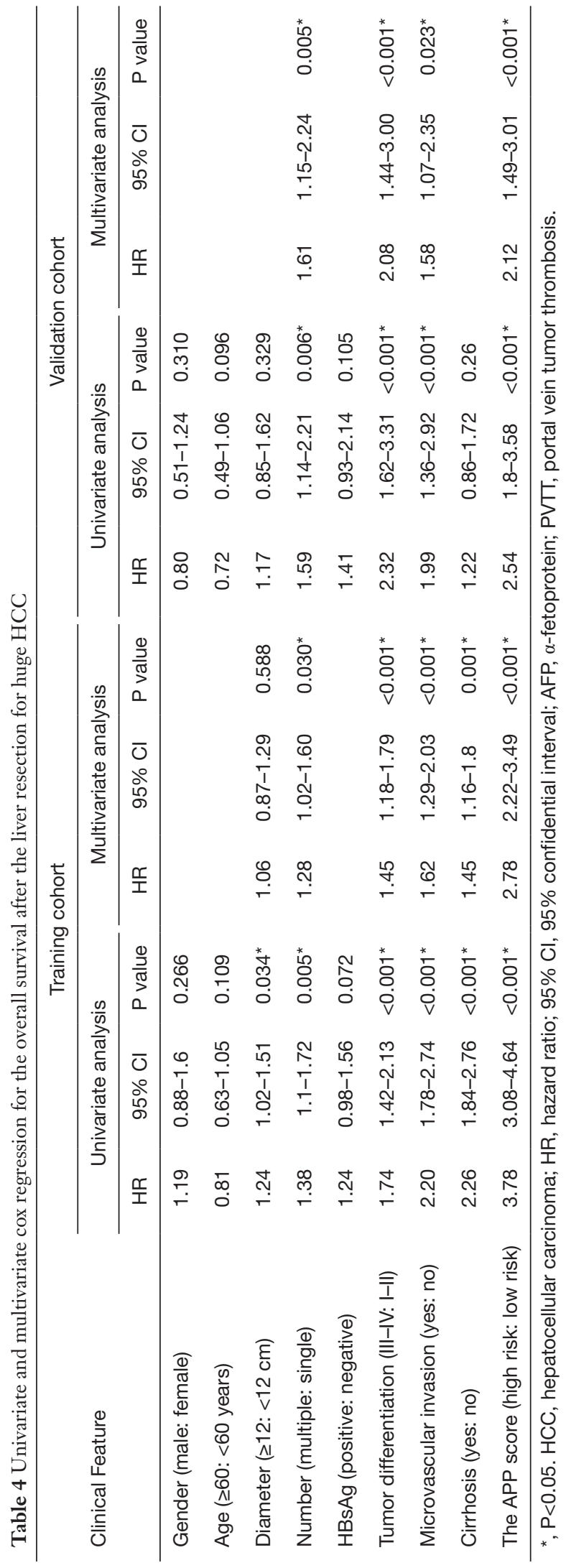

In this study, three independent risk factors for postoperative 90-day mortality were identified for huge HCC patients: AFP levels, prealbumin levels, and PVTT status. A nomogram model (APP score) was constructed and validated using two independent cohorts to accurately estimate the risk of postoperative 90 -day mortality for huge HCC patients before hepatectomy. Moreover, the APP score showed greater predictive value for 90-day mortality and long-term survival than the Child-Pugh, MELD, and ALBI scores. Derived from only three preoperatively available parameters, this nomogram-based model can be easily adapted by surgeons for patient selection and for optimizing treatment for patients with huge HCC in the future.

Postoperative liver failure and tumor recurrence were major contributors to 90-day mortality $(38,39)$, the related preoperative factors were incorporated into the predictive model. Prealbumin levels were reported as an independent predictor of liver failure after hepatectomy (40). With a short half-life ( $<48$ hours) and resistance to interference by extraneous supplements, serum prealbumin is a sensitive marker for liver function (41). AFP levels and PVTT status could reflect tumor status, which widely accepted that a high serum AFP level (42) and the presence of PVTT (43) indicate a poor prognosis for HCC patients. Moreover, the presence of PVTT makes liver resection more techniquedemanding (44). For a patient with PVTT, hepatectomy is recommended to en bloc removal of the tumor thrombosis or to extract the tumor thrombus through the stump of a portal vein branch. Thus, massive intraoperative bleeding may result because both the portal and hepatic veins are vulnerable to injury during such procedures $(45,46)$.

In the current study, the 90-day mortality in patients with huge HCC accompanied by PVTT was significantly higher than in patients without PVTT $(22.8 \%$ vs. $4.1 \%, \mathrm{P}<0.001)$. The mortality rates both in the patients with PVTT and without PVTT were consistent with findings from the previous literature $(8,9,45,46)$. The proportion of patients with PVTT might play a major role in the total mortality rate. Intriguingly, an interaction between prealbumin and PVTT was observed in the logistic regression analysis. After analyzing the nomogram, the protective effect of the prealbumin seemed to be obliterated by the presence of PVTT. We speculate that the presence of PVTT could decrease liver blood flow and impair functional liver reserve, leading to a catabolic state and the synthesis of less prealbumin (47), which could explain the interaction between PVTT and prealbumin. 
Our model performed well in predicting 90-day mortality for patients with huge HCC who underwent liver resection in the training and validation cohort. Moreover, an independent prospective cohort also confirmed our finding, indicating the robustness of the model. Because some well-established preoperative model had been proven to be effective in predicting the morbidity and mortality after liver resection for HCC $(13-16,19,48,49)$, we compared the APP score with the Child-Pugh, MELD, and ALBI scores. We found that APP score showed improved accuracy compared with three conventional preoperative assessment models (Child-Pugh, MELD, and ALBI scores) in predicting postoperative 90-day mortality of huge HCC patients with liver resection. The poor performance of conventional models may be explained by the fact that they were not specifically designed to assess 90 -day mortality or predict fatal tumor recurrence.

The APP score could stratify the candidates of liver resection for huge HCC into two different risk groups for postoperative 90-day mortality. Based on this risk evaluation, we can provide individualized treatment recommendations for these patients. In the whole retrospective cohort, the high-risk group (APP score >-1.96) consisted of only $24.4 \%$ of the entire retrospective cohort but accounted for $80.4 \%$ of those who died within 90 days after surgery (Figure S2). As postoperative 90-day mortality was greater than $30 \%$ in the high-risk group, we do not recommend liver resection for this subgroup; and targeted therapy, TACE, or radiotherapy may be better choices instead. A low incidence of 90 -day mortality $(\sim 2 \%)$ was observed in the low-risk group, similar to that of patients with common HCC who underwent routine hepatectomy (50). Thus, patients with huge HCC categorized in the low-risk group (APP score $\leq-1.96$ ) can be considered suitable candidates for hepatectomy. Although the accuracy for predicting the risk of 90-day mortality by the APP score still needs further improvement, we believed that the classification of highand low-risk groups could be helpful to avoid futile liver resection and choose optimized treatment.

There are a few limitations of this study. The predictive model requires further validation in a larger multicenter study. Meanwhile, our patients were all from China, and most had a history of HBV infection. Therefore, whether the APP score is applicable to patients of a Western nationality with huge HCC should be explored. In addition, indocyanine green tests and ultrasound elastography (FibroScan) were not routinely performed at our institute until 2014. Thus, we did not include these two parameters in the current study.

In conclusion, we established a novel predictive model (the APP score) to precisely predict the risk of postoperative 90-day mortality for patients with huge HCC undergoing hepatectomy. Furthermore, the predictive capability of the APP score in terms of 90-day mortality and OS is superior to the Child-Pugh score, the MELD score, and the ALBI score. The APP score may thus serve as a valuable tool for surgeons to identify suitable candidates for liver resection so as to improve the safety and efficacy of surgical treatments for huge HCC patients.

\section{Acknowledgments}

Funding: This study was supported by grants from the National Key R\&D Program of China (2019YFC1315800, 2019YFC1315802), the National Key Research and Development Program (2016YFF0101405), the State Key Program of National Natural Science of China (81830102), the National Natural Science Foundation of China (81772578, 81772551, 81872355, 81802991, and 82072715), the Shanghai Municipal Health Commission Collaborative Innovation Cluster Project (2019CXJQ02), Projects from the Shanghai Science and Technology Commission (19441905000), Shanghai "Rising Stars of Medical Talent" Youth Development Program (Outstanding Youth Medical Talents) and Projects from Shanghai Municipal Key Clinical Specialty.

\section{Footnote}

Reporting Checklist: The authors have completed the STROBE reporting checklist. Available at http://dx.doi. org/10.21037/atm-20-7842

Data Sharing Statement: Available at http://dx.doi. org/10.21037/atm-20-7842

Conflicts of Interest: All authors have completed the ICMJE uniform disclosure form (available at http://dx.doi. org/10.21037/atm-20-7842). The authors have no conflicts of interest to declare. None of the author serves as a current Editorial Team member for this journal.

Ethical Statement: The authors are accountable for all aspects of the work in ensuring that questions related to the accuracy or integrity of any part of the work are appropriately investigated and resolved. The study was 
conducted in accordance with the Declaration of Helsinki (as revised in 2013). Ethical approval for the use of patient information was obtained from the Zhongshan Hospital Research Ethics Committee (No. B2021-017R). Informed consent was obtained from all patients.

Open Access Statement: This is an Open Access article distributed in accordance with the Creative Commons Attribution-NonCommercial-NoDerivs 4.0 International License (CC BY-NC-ND 4.0), which permits the noncommercial replication and distribution of the article with the strict proviso that no changes or edits are made and the original work is properly cited (including links to both the formal publication through the relevant DOI and the license). See: https://creativecommons.org/licenses/by-nc-nd/4.0/.

\section{References}

1. Siegel RL, Miller KD, Jemal A. Cancer statistics, 2018. CA Cancer J Clin 2018;68:7-30.

2. Pawlik TM, Poon RT, Abdalla EK, et al. Critical appraisal of the clinical and pathologic predictors of survival after resection of large hepatocellular carcinoma. Arch Surg 2005;140:450-7; discussion 457-8.

3. Chang YJ, Chung KP, Chang YJ, et al. Long-term survival of patients undergoing liver resection for very large hepatocellular carcinomas. Br J Surg 2016;103:1513-20.

4. Glantzounis GK, Paliouras A, Stylianidi MC, et al. The role of liver resection in the management of intermediate and advanced stage hepatocellular carcinoma. A systematic review. Eur J Surg Oncol 2018;44:195-208.

5. Takamoto T, Sugawara Y, Hashimoto T, et al. Evaluating the current surgical strategies for hepatocellular carcinoma. Expert Rev Gastroenterol Hepatol 2016;10:341-57.

6. Chen H, Jia W. Progress in hepatectomy for hepatocellular carcinoma and peri-operation management. Genes Dis 2020;7:320-7.

7. Gunasekaran G, Bekki Y, Lourdusamy V, et al. Surgical Treatments of Hepatobiliary Cancers. Hepatology 2021;73 Suppl 1:128-36.

8. Lim C, Compagnon P, Sebagh M, et al. Hepatectomy for hepatocellular carcinoma larger than $10 \mathrm{~cm}$ : preoperative risk stratification to prevent futile surgery. HPB (Oxford) 2015;17:611-23.

9. Zheng J, Shen S, Jiang L, et al. Outcomes of anterior approach major hepatectomy with diaphragmatic resection for single huge right lobe HCC with diaphragmatic invasion. Medicine (Baltimore) 2018;97:e12194.

10. Goh BK, Kam JH, Lee SY, et al. Significance of neutrophil-to-lymphocyte ratio, platelet-to-lymphocyte ratio and prognostic nutrition index as preoperative predictors of early mortality after liver resection for huge $(>/=10 \mathrm{~cm})$ hepatocellular carcinoma. J Surg Oncol 2016;113:621-7.

11. Khalaf N, Ying J, Mittal S, et al. Natural History of Untreated Hepatocellular Carcinoma in a US Cohort and the Role of Cancer Surveillance. Clin Gastroenterol Hepatol 2017;15:273-81.e1.

12. Li C, Shen JY, Zhang XY, et al. Predictors of Futile Liver Resection for Patients with Barcelona Clinic Liver Cancer Stage B/C Hepatocellular Carcinoma. J Gastrointest Surg 2018;22:496-502.

13. Kok B, Abraldes JG. Child-Pugh Classification: Time to Abandon? Semin Liver Dis 2019;39:96-103.

14. Teh SH, Christein J, Donohue J, et al. Hepatic resection of hepatocellular carcinoma in patients with cirrhosis: Model of End-Stage Liver Disease (MELD) score predicts perioperative mortality. J Gastrointest Surg 2005;9:120715; discussion 1215 .

15. Serenari M, Han K, Ravaioli F, et al. A nomogram based on liver stiffness predicts postoperative complications in patients with hepatocellular carcinoma. J Hepatol 2020;73:855-62.

16. Johnson PJ, Berhane S, Kagebayashi C, et al. Assessment of liver function in patients with hepatocellular carcinoma: a new evidence-based approach-the ALBI grade. J Clin Oncol 2015;33:550-8.

17. Kokudo T, Hasegawa K, Shirata C, et al. Assessment of Preoperative Liver Function for Surgical Decision Making in Patients with Hepatocellular Carcinoma. Liver cancer 2019;8:447-56.

18. Fagenson AM, Gleeson EM, Pitt HA, et al. AlbuminBilirubin Score vs Model for End-Stage Liver Disease in Predicting Post-Hepatectomy Outcomes. J Am Coll Surg 2020;230:637-45.

19. Ross SW, Seshadri R, Walters AL, et al. Mortality in hepatectomy: Model for End-Stage Liver Disease as a predictor of death using the National Surgical Quality Improvement Program database. Surgery 2016;159:777-92.

20. Wang YY, Zhong JH, Su ZY, et al. Albumin-bilirubin versus Child-Pugh score as a predictor of outcome after liver resection for hepatocellular carcinoma. Br J Surg 2016;103:725-34.

21. Zaydfudim VM, Turrentine FE, Smolkin ME, et al. The 
impact of cirrhosis and MELD score on postoperative morbidity and mortality among patients selected for liver resection. Am J Surg 2020;220:682-6.

22. Durand F, Valla D. Assessment of the prognosis of cirrhosis: Child-Pugh versus MELD. J Hepatol 2005;42 Suppl 1:S100-7.

23. Simons JP, Hill JS, Ng SC, et al. Perioperative mortality for management of hepatic neoplasm: a simple risk score. Ann Surg 2009;250:929-34.

24. Herbert GS, Prussing KB, Simpson AL, et al. Early trends in serum phosphate and creatinine levels are associated with mortality following major hepatectomy. HPB (Oxford) 2015;17:1058-65.

25. Hsu HY, Yu MC, Lee CW, et al. RAM score is an effective predictor for early mortality and recurrence after hepatectomy for hepatocellular carcinoma. BMC Cancer 2017;17:742.

26. Gyoeri GP, Pereyra D, Braunwarth E, et al. The 3-60 criteria challenge established predictors of postoperative mortality and enable timely therapeutic intervention after liver resection. Hepatobiliary Surg Nutr 2019;8:111-24.

27. Sahara K, Tsilimigras DI, Paredes AZ, et al. Development and validation of a real-time mortality risk calculator before, during and after hepatectomy: an analysis of the ACS NSQIP database. HPB (Oxford) 2020;22:1158-67.

28. Dasari BVM, Hodson J, Sutcliffe RP, et al. Developing and validating a preoperative risk score to predict 90-day mortality after liver resection. J Surg Oncol 2019;119:472-8.

29. Cheng S, Chen M, Cai J, et al. Chinese Expert Consensus on Multidisciplinary Diagnosis and Treatment of Hepatocellular Carcinoma with Portal Vein Tumor Thrombus (2018 Edition). Liver cancer 2020;9:28-40.

30. Bezerra AS, D'Ippolito G, Faintuch S, et al. Determination of splenomegaly by CT: is there a place for a single measurement? AJR Am J Roentgenol 2005;184:1510-3.

31. Llovet JM, Brú C, Bruix J. Prognosis of hepatocellular carcinoma: the BCLC staging classification. Semin Liver Dis 1999; 19:329-38.

32. Urata K, Hashikura Y, Ikegami T, et al. Standard liver volume in adults. Transplant Proc 2000;32:2093-4.

33. Zhou J, Sun HC, Wang Z, et al. Guidelines for Diagnosis and Treatment of Primary Liver Cancer in China (2017 Edition). Liver Cancer 2018;7:235-60.

34. Strasberg SM. Nomenclature of hepatic anatomy and resections: a review of the Brisbane 2000 system. J Hepatobiliary Pancreat Surg 2005;12:351-5.

35. Dindo D, Demartines N, Clavien PA. Classification of surgical complications: a new proposal with evaluation in a cohort of 6336 patients and results of a survey. Ann Surg 2004;240:205-13.

36. Ren ZG, Lin ZY, Xia JL, et al. Postoperative adjuvant arterial chemoembolization improves survival of hepatocellular carcinoma patients with risk factors for residual tumor: a retrospective control study. World J Gastroenterol 2004;10:2791-4.

37. Rahbari NN, Garden OJ, Padbury R, et al. Posthepatectomy liver failure: a definition and grading by the International Study Group of Liver Surgery (ISGLS). Surgery 2011;149:713-24.

38. Paugam-Burtz C, Janny S, Delefosse D, et al. Prospective validation of the "fifty-fifty" criteria as an early and accurate predictor of death after liver resection in intensive care unit patients. Ann Surg 2009;249:124-8.

39. Kim BW, Kim YB, Wang HJ, et al. Risk factors for immediate post-operative fatal recurrence after curative resection of hepatocellular carcinoma. World J Gastroenterol 2006;12:99-104.

40. Huang L, Li J, Yan JJ, et al. Prealbumin is predictive for postoperative liver insufficiency in patients undergoing liver resection. World J Gastroenterol 2012;18:7021-5.

41. Dellière $S$, Cynober L. Is transthyretin a good marker of nutritional status? Clin Nutr 2017;36:364-70.

42. Yang SL, Liu LP, Yang S, et al. Preoperative serum alpha-fetoprotein and prognosis after hepatectomy for hepatocellular carcinoma. Br J Surg 2016;103:716-24.

43. Shi J, Lai EC, Li N, et al. Surgical treatment of hepatocellular carcinoma with portal vein tumor thrombus. Ann Surg Oncol 2010;17:2073-80.

44. Zhang XP, Gao YZ, Chen ZH, et al. An Eastern Hepatobiliary Surgery Hospital/Portal Vein Tumor Thrombus Scoring System as an Aid to Decision Making on Hepatectomy for Hepatocellular Carcinoma Patients With Portal Vein Tumor Thrombus: A Multicenter Study. Hepatology 2019;69:2076-90.

45. Kamiyama T, Kakisaka T, Orimo T, et al. Hepatectomy for hepatocellular carcinoma with portal vein tumor thrombus. World J Hepatol 2017;9:1296-304.

46. Chok KS, Cheung TT, Chan SC, et al. Surgical outcomes in hepatocellular carcinoma patients with portal vein tumor thrombosis. World J Surg 2014;38:490-6.

47. Beck FK, Rosenthal TC. Prealbumin: a marker for nutritional evaluation. Am Fam Physician 2002;65:1575-8.

48. Kong FH, Miao XY, Zou H, et al. End-stage liver disease score and future liver remnant volume predict post- 
hepatectomy liver failure in hepatocellular carcinoma. World J Clin Cases 2019;7:3734-41.

49. Chin KM, Allen JC, Teo JY, et al. Predictors of posthepatectomy liver failure in patients undergoing extensive liver resections for hepatocellular carcinoma. Ann

Cite this article as: Yin Y, Cheng JW, Chen FY, Chen XX, Zhang X, Huang A, Guo DZ, Wang YP, Cao Y, Fan J, Zhou J, Yang XR. A novel preoperative predictive model of 90day mortality after liver resection for huge hepatocellular carcinoma. Ann Transl Med 2021;9(9):774. doi: 10.21037/atm$20-7842$
Hepatobiliary Pancreat Surg 2018;22:185-96.

50. Hughes MJ, McNally S, Wigmore SJ. Enhanced recovery following liver surgery: a systematic review and metaanalysis. HPB (Oxford) 2014;16:699-706. 

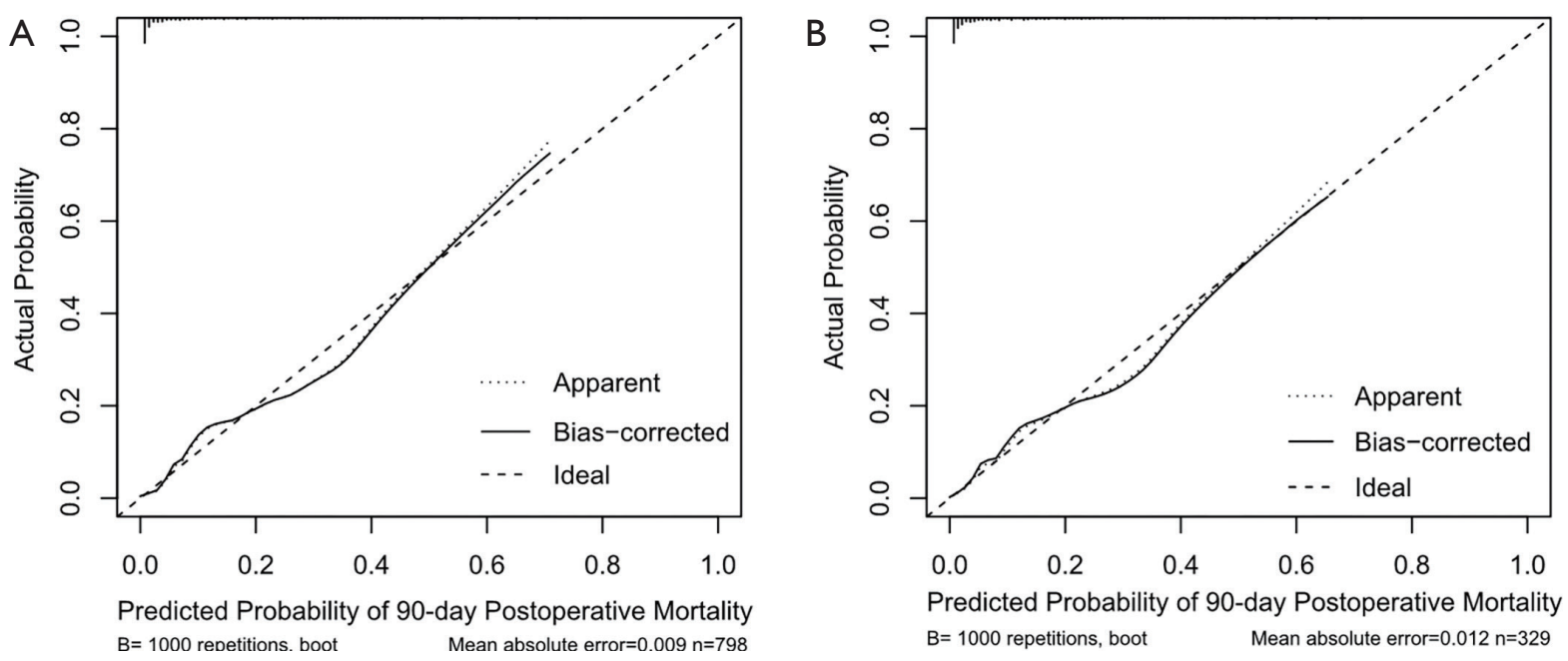

Figure S1 Calibration Curve by bootstrap method of the model. The apparent and the bias-corrected curves showed good agreement with the ideal curve in both (A) training cohort, and (B) validation cohort.

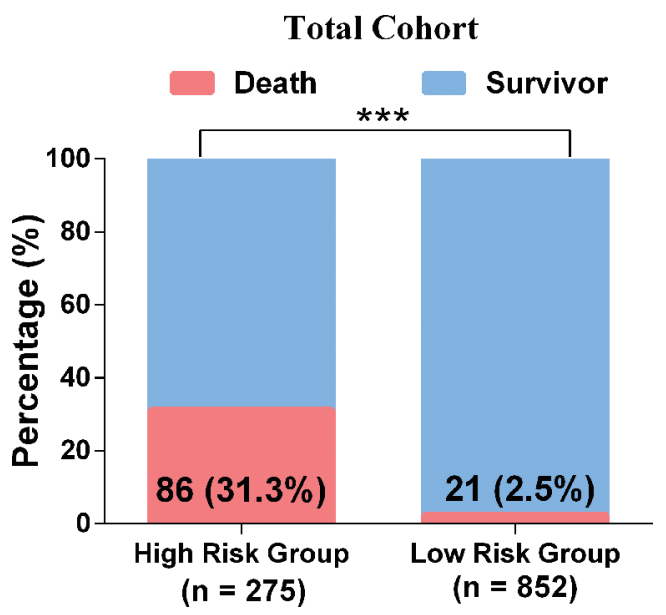

Figure $\mathbf{S} 2$ The predictive performance of the APP score for the postoperative 90-day mortality for huge HCC in the entire retrospective cohort. In the entire cohort, the higher incidence of 90-day mortality after hepatectomy was observed in the highrisk group (APP score >-1.96) compared with that of the low-risk group (APP score $\leq-1.96),\left(31.3 \%\right.$ vs. $\left.2.5 \%,{ }^{* * *} \mathrm{P}<0.001\right)$. 
Table S1 Detailed clinicopathologic characteristics of the training, validation, and prospective cohorts

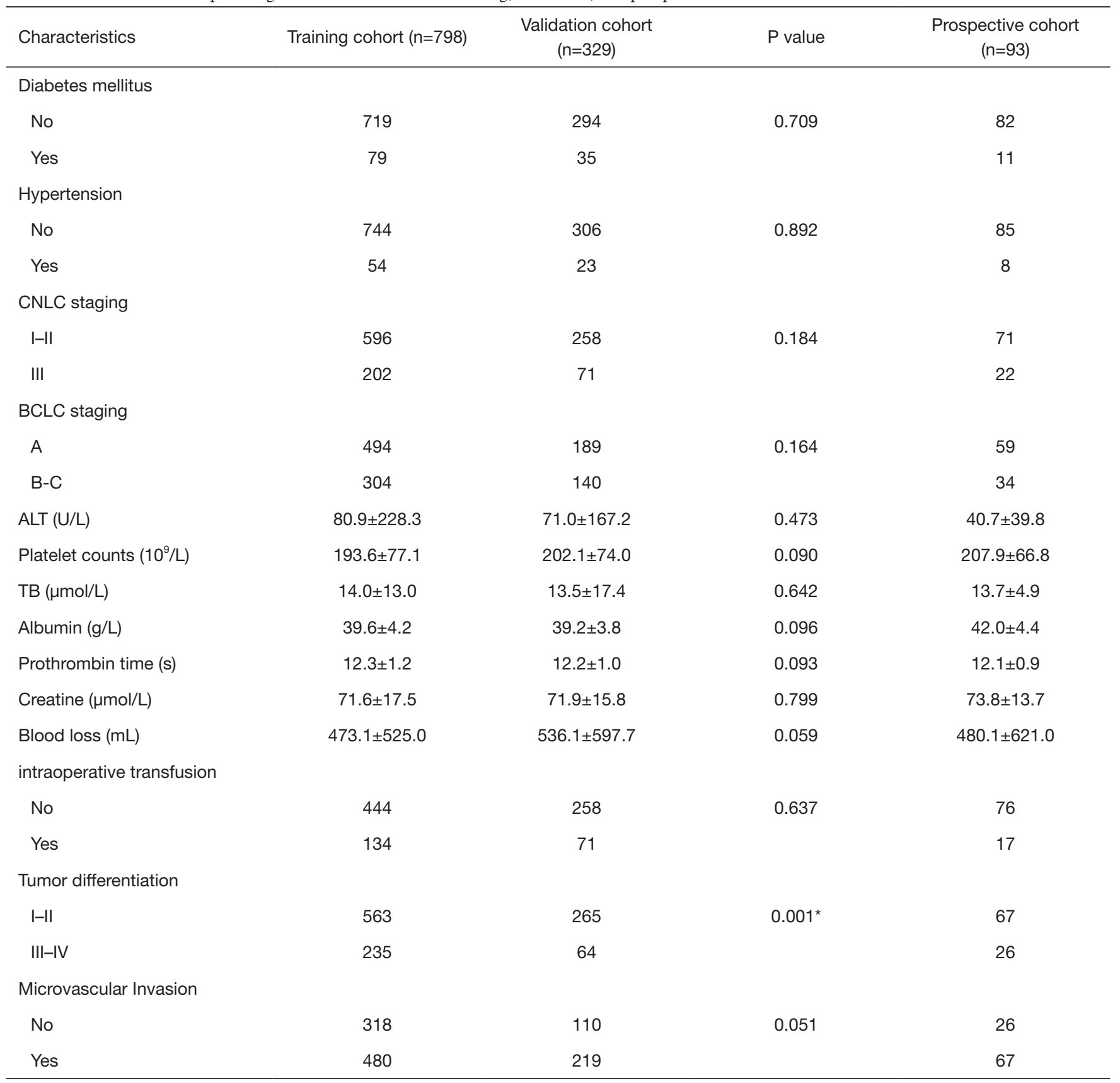

CNLC staging, China liver cancer staging; BCLC staging, Barcelona clinic liver cancer staging; GGT, $\gamma$-glutamyl transpeptidase; ALT, alanine aminotransferase; TB, total bilirubin. 
Table S2 The subgroup analysis of APP score in the 90-day mortality in training and validation cohort

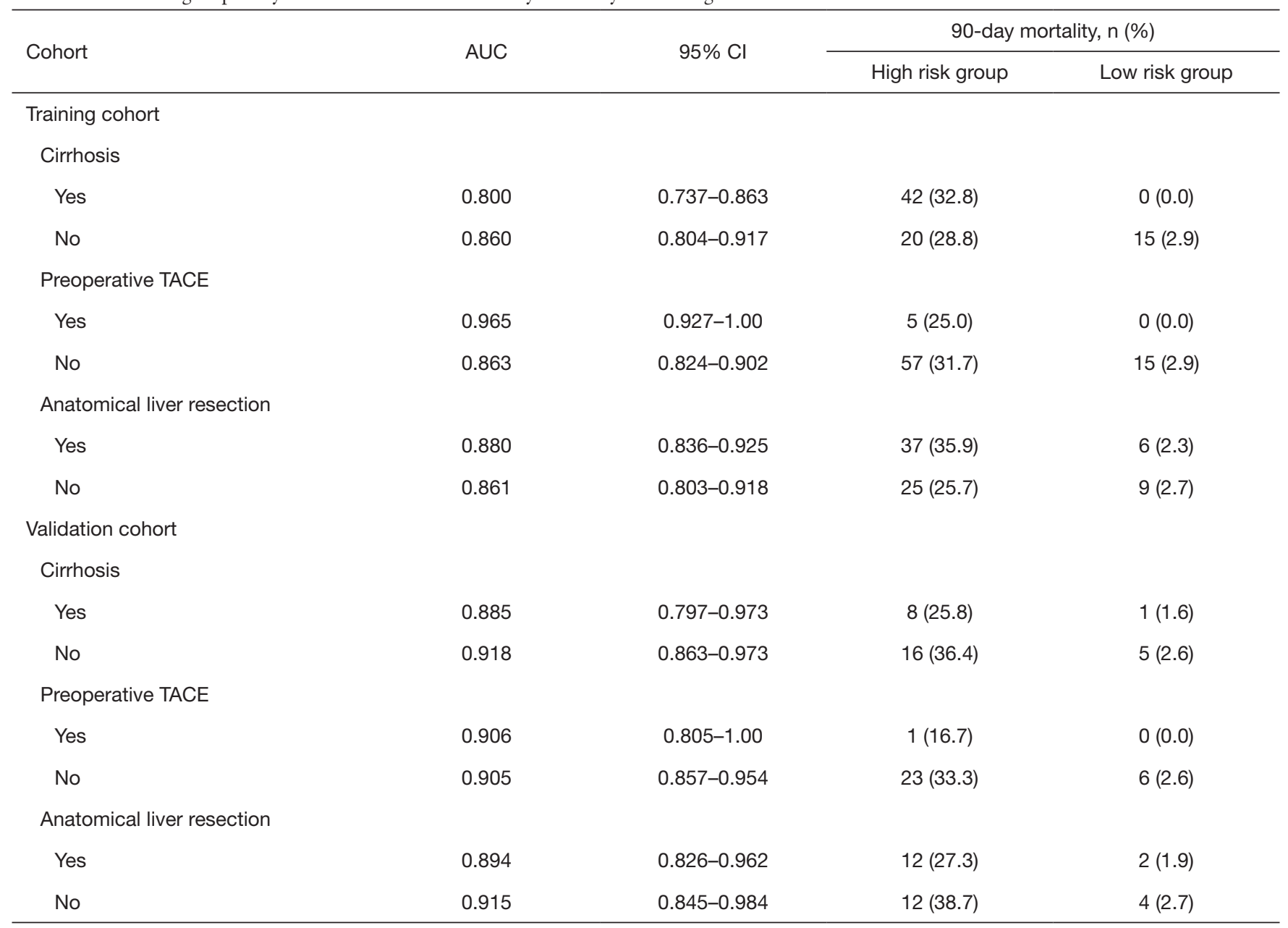

AUC, area under curve; 95\% Cl, 95\% confidential interval; TACE, transcatheter arterial chemoembolization. 